Research Article

\title{
Existence of the Solution for System of Coupled Hybrid Differential Equations with Fractional Order and Nonlocal Conditions
}

\begin{abstract}
Khalid Hilal and Ahmed Kajouni
Laboratoire de Mathématiques Appliquées \& Calcul Scientifique, Université Sultan Moulay Slimane, BP 523,
\end{abstract} 23000 Béni Mellal, Morocco

Correspondence should be addressed to Ahmed Kajouni; kajjouni@gmail.com

Received 10 February 2016; Revised 29 April 2016; Accepted 3 May 2016

Academic Editor: Gaston Mandata N'guérékata

Copyright (c) $2016 \mathrm{~K}$. Hilal and A. Kajouni. This is an open access article distributed under the Creative Commons Attribution License, which permits unrestricted use, distribution, and reproduction in any medium, provided the original work is properly cited.

This paper is motivated by some papers treating the fractional hybrid differential equations with nonlocal conditions and the system of coupled hybrid fractional differential equations; an existence theorem for fractional hybrid differential equations involving Caputo differential operators of order $1<\alpha \leq 2$ is proved under mixed Lipschitz and Carathéodory conditions. The existence and uniqueness result is elaborated for the system of coupled hybrid fractional differential equations.

\section{Introduction}

Our aim in this paper is to study the existence of solution for the boundary value problems for hybrid differential equations with fractional order $1<\alpha \leq 2$ and nonlocal condition (BVPHDEFNL for short) of the form

$$
\begin{aligned}
{ }^{c} D^{\alpha}\left(\frac{x(t)}{f(t, x(t))}\right) & =g(t, x(t)) \\
& \text { a.e. } t \in J=[0, T], 1<\alpha \leq 2, \\
\frac{x(0)}{f(0, x(0))} & =\mathscr{L}(x), \\
\frac{x(T)}{f(T, x(T))} & =x_{T},
\end{aligned}
$$

where ${ }^{c} D^{\alpha}$ is the Caputo fractional derivative.

$f \in C(J \times \mathbb{R}, \mathbb{R} \backslash\{0\}), g \in \mathscr{C}(J \times \mathbb{R}, \mathbb{R}), \mathscr{L}: C(J, \mathbb{R}) \rightarrow$ $\mathbb{R}$ is a continuous function and $x_{T} \in \mathbb{R}$. And exploitation of results obtained to study the existence of solutions for a system of coupled hybrid fractional differential equations is as follows:

$$
\begin{aligned}
{ }^{c} D^{\alpha}\left(\frac{x(t)}{f_{1}(t, x(t), y(t))}\right)= & g_{1}(t, x(t), y(t)) \\
& \text { a.e. } t \in[0,1], 1<\alpha \leq 2, \\
{ }^{c} D^{\beta}\left(\frac{y(t)}{f_{2}(t, x(t), y(t))}\right)= & g_{2}(t, x(t), y(t)) \\
& \text { a.e. } t \in[0,1], 1<\beta \leq 2, \\
\frac{x(0)}{f_{1}(0, x(0), y(0))}= & \mathscr{L}_{1}(x, y) ; \quad x(1)=0, \\
\frac{y(0)}{f_{2}(0, x(0), y(0))}= & \mathscr{L}_{2}(x, y) ; \quad y(1)=0,
\end{aligned}
$$

where ${ }^{c} D^{\alpha}$ is the Caputo fractional derivative.

$f_{i} \in C([0,1] \times \mathbb{R} \times \mathbb{R}, \mathbb{R} \backslash\{0\}), g_{i} \in C([0,1] \times \mathbb{R} \times \mathbb{R}, \mathbb{R})$, and $\mathscr{L}_{i}: C([0,1], \mathbb{R}) \times C([0,1], \mathbb{R}) \rightarrow \mathbb{R}$ are continuous functions $(i=1,2)$. 
Fractional differential equations are a generalization of ordinary differential equations and integration to arbitrary noninteger orders. The origin of fractional calculus goes back to Newton and Leibniz in the seventeenth century. It is widely and efficiently used to describe many phenomena arising in engineering, physics, economy, and science. There are several concepts of fractional derivatives, some classical, such as Riemann-Liouville or Caputo definitions. For noteworthy papers dealing with the integral operator and the arbitrary fractional order differential operator, see [1-7].

The quadratic perturbations of nonlinear differential equations have attracted much attention. We call such fractional hybrid differential equations. There have been many works on the theory of hybrid differential equations, and we refer the readers to the articles [8-12].

Dhage and Lakshmikantham [11] discussed the following first order hybrid differential equation

$$
\begin{aligned}
\frac{d}{d t}\left[\frac{x(t)}{f(t, x(t))}\right] & =g(t, x(t)) \quad \text { a.e. } t \in J=[0, T], \\
x\left(t_{0}\right) & =x_{0} \in \mathbb{R},
\end{aligned}
$$

where $f \in C(J \times \mathbb{R}, \mathbb{R} \backslash\{0\})$ and $g \in \mathscr{C}(J \times \mathbb{R}, \mathbb{R})$. They established the existence, uniqueness results, and some fundamental differential inequalities for hybrid differential equations initiating the study of theory of such systems and proved, utilizing the theory of inequalities, the existence of extremal solutions and comparison results.

Zhao et al. [13] have discussed the following fractional hybrid differential equations involving Riemann-Liouville differential operators:

$$
\begin{aligned}
D^{q}\left[\frac{x(t)}{f(t, x(t))}\right] & =g(t, x(t)) \quad \text { a.e. } t \in J=[0, T], \\
x(0) & =0,
\end{aligned}
$$

where $f \in C(J \times \mathbb{R}, \mathbb{R} \backslash\{0\})$ and $g \in \mathscr{C}(J \times \mathbb{R}, \mathbb{R})$. The authors of [13] established the existence theorem for fractional hybrid differential equation and some fundamental differential inequalities. They also established the existence of extremal solutions.

Hilal and Kajouni [14] have studied boundary fractional hybrid differential equations involving Caputo differential operators of order $0<\alpha<1$ as follows:

$$
\begin{aligned}
& D^{\alpha}\left(\frac{x(t)}{f(t, x(t))}\right)=g(t, x(t)) \\
& \text { a.e. } t \in J=[0, T] \text {, } \\
& a \frac{x(0)}{f(0, x(0))}+b \frac{x(T)}{f(T, x(T))}=c,
\end{aligned}
$$

where $f \in C(J \times \mathbb{R}, \mathbb{R} \backslash\{0\}), g \in \mathscr{C}(J \times \mathbb{R}, \mathbb{R})$ and $a, b$, and $c$ are real constants with $a+b \neq 0$. They proved the existence result for boundary fractional hybrid differential equations under mixed Lipschitz and Carathéodory conditions. Some fundamental fractional differential inequalities are also established which are utilized to prove the existence of extremal solutions. Necessary tools are considered and the comparison principle is proved which will be useful for further study of qualitative behavior of solutions.

The nonlocal condition is a condition attached to the main equation; it replaces the classic nonlocal condition in order to model physical phenomena of the fashion nearest from reality. The nonlocal condition involves the function

$$
\mathscr{L}(x)=\sum_{i=1}^{p} c_{i} x\left(t_{i}\right)
$$

where $c_{i}, i=1,2, \ldots, p$, are given constants and $0<t_{1}<t_{2}<$ $\cdots<t_{p}$.

Let us observe that Cauchy problems with nonlocal conditions were initiated by Byszewski and Lakshmikantham [2] and, since then, such problems have also attracted several authors including A. Aizicovici, K. Ezzinbi, Z. Fan, J. Liu, J. Liang, Y. Lin, T.-J. Xiao, G. N’Guérékata, E. Hernàndez, and H. Lee (see $[2,15])$.

\section{Preliminaries}

In this section, we introduce notations, definitions, and preliminary facts which are used throughout this paper.

By $X=C(J, \mathbb{R})$ we denote the Banach space of all continuous functions from $J=[0, T]$ into $\mathbb{R}$ with the norm

$$
\|y\|=\sup \{|y(t)|, t \in J\} .
$$

And let $\mathscr{C}(J \times \mathbb{R}, \mathbb{R})$ denote the class of functions $g: J \times \mathbb{R} \rightarrow$ $\mathbb{R}$ such that

(i) the map $t \mapsto g(t, x)$ is measurable for each $x \in \mathbb{R}$,

(ii) the map $x \mapsto g(t, x)$ is continuous for each $t \in J$.

The class $\mathscr{C}(J \times \mathbb{R}, \mathbb{R})$ is called the Carathéodory class of functions on $J \times \mathbb{R}$ which are Lebesgue integrable when bounded by a Lebesgue integrable function on $J$.

By $L^{1}(J ; \mathbb{R})$ we denote the space of Lebesgue integrable real-valued functions on $J$ equipped with the norm $\|\cdot\|_{L^{1}}$ defined by

$$
\|x\|_{L^{1}}=\int_{0}^{T}|x(s)| d s .
$$

Definition 1. The fractional integral of the function $h \in$ $L^{1}\left([a, b], \mathbb{R}_{+}\right)$of order $\alpha \in \mathbb{R}_{+}$is defined by

$$
I_{a}^{\alpha} h(t)=\frac{1}{\Gamma(\alpha)} \int_{a}^{t}(t-s)^{\alpha-1} h(s) d s,
$$

where $\Gamma$ is the gamma function.

Definition 2. For a function $h$ given on the interval $[a, b]$, the Caputo fractional order derivative of $h$ is defined by

$$
{ }^{c} D_{a^{+}}^{\alpha} h(t)=\frac{1}{\Gamma(n-\alpha)} \int_{a}^{t}(t-s)^{n-\alpha-1} h^{(n)}(s) d s,
$$

where $n=[\alpha]+1$ and $[\alpha]$ denotes the integer part of $\alpha$. 
Lemma 3 (see [16]). Let $\alpha>0$. Then the fractional differential equation

$$
{ }^{c} D^{\alpha} h(t)=0
$$

has solutions

$$
\begin{aligned}
& h(t)=c_{0}+c_{1} t+\cdots+c_{n-1} t^{n-1}, \\
& \qquad c_{i} \in \mathbb{R}, i=1,2, \ldots, n-1, n=[\alpha]+1 .
\end{aligned}
$$

Lemma 4 (see $[16])$. Let $\alpha>0$. Then

$$
I^{\alpha} D^{\alpha} h(t)=h(t)+c_{0}+c_{1} t+\cdots+c_{n-1} t^{n-1},
$$

for some $c_{i} \in \mathbb{R}, i=1,2, \ldots, n-1, n=[\alpha]+1$.

Definition 5. By a solution of the BVPHDEFNL (1) we mean a function $x \in C(J, \mathbb{R})$ such that

(i) $d^{2} u / d t^{2} \in L^{1}(J, \mathbb{R})$, where $u: t \mapsto x / f(t, x)$ for each $x \in \mathbb{R}$,

(ii) $x$ satisfies the equations in (1).

\section{Existence Result}

In this section, we prove the existence results for the boundary value problems for hybrid differential equations with fractional order (1) on the closed and bounded interval $J=$ $[0, T]$ under mixed Lipschitz and Carathéodory conditions on the nonlinearities involved in it.

We defined the multiplication in $X$ by

$$
(x y)(t)=x(t) y(t), \quad \text { for } x, y \in X .
$$

Clearly, $X=C(J ; \mathbb{R})$ is a Banach algebra with respect to above norm and multiplication in it.

We prove the existence of solution for the BVPHDEFNL (1) by a fixed point theorem in Banach algebra due to Dhage [10].

Lemma 6 (see [10]). Let $S$ be a nonempty, closed convex, and bounded subset of the Banach algebra $X$ and let $A: X \rightarrow X$ and $B: X \rightarrow X$ be two operators such that

(a) A is Lipschitzian with a Lipschitz constant $\alpha$,

(b) $B$ is completely continuous,

(c) $x=A x B y \Rightarrow x \in S$ for all $y \in S$,

(d) $\alpha M<1$, where $M=\|B(S)\|=\sup \{\|B(x)\|: x \in S\}$.

Then the operator equation $A x B x=x$ has a solution in $S$.

We make the following assumptions:

$\left(\mathrm{H}_{0}\right)$ : the function $x \mapsto x / f(t, x)$ is increasing in $\mathbb{R}$ almost everywhere for $t \in J$.

$\left(\mathrm{H}_{1}\right)$ : there exists a constant $L>0$ such that

$$
|f(t, x)-f(t, y)| \leq L|x-y|
$$

for all $t \in J$ and $x, y \in \mathbb{R}$.
$\left(\mathrm{H}_{2}\right)$ : there exists a function $h \in L^{1}(J, \mathbb{R})$ such that

$$
|g(t, x)| \leq h(t) \quad \text { a.e. } t \in J
$$

for all $x \in \mathbb{R}$.

$\left(\mathrm{H}_{3}\right)$ : there exists a constant $M>0$ such that $|\mathscr{L}(y)| \leq M$, for each $y \in C(J ; \mathbb{R})$.

As a consequence of Lemmas 3 and 4 we have the following result which is useful in what follows.

Lemma 7. Assume that hypothesis $\left(H_{0}\right)$ holds. Then for any $h \in L^{1}(J ; \mathbb{R})$, the function $x \in C(J ; \mathbb{R})$ is a solution of the BVPHDEFNL:

$$
{ }^{c} D^{\alpha}\left(\frac{x(t)}{f(t, x(t))}\right)=h(t)
$$

$$
\text { a.e. } t \in J=[0, T], 1<\alpha \leq 2 \text {, }
$$

$$
\frac{x(0)}{f(0, x(0))}=\mathscr{L}(x) \text {, }
$$

$$
\frac{x(T)}{f(T, x(T))}=x_{T}
$$

if and only if $x$ satisfies the hybrid integral equation

$$
\begin{aligned}
x(t) & =f(t, x(t))\left[\frac{1}{\Gamma(\alpha)} \int_{0}^{t}(t-s)^{\alpha-1} h(s) d s\right. \\
& -\frac{t}{T \Gamma(\alpha)} \int_{0}^{T}(T-s)^{\alpha-1} h(s) d s-\left(\frac{t}{T}-1\right) \mathscr{L}(x) \\
& \left.+\frac{t}{T} x_{T}\right] .
\end{aligned}
$$

Proof. Assume that $x$ is a solution of the problem (18). Applying the Caputo fractional operator of the order $\alpha$, we obtain the first equation in (17). Again, substituting $t=0$ and $t=T$ in (18) we will have the second equation in (17).

Conversely, ${ }^{c} D^{\alpha}(x(t) / f(t, x(t)))=h(t)$, so we get

$$
\frac{x(t)}{f(t, x(t))}+c_{0}+c_{1} t=I^{\alpha} h(t) .
$$

Then $x(0) / f(0, x(0))+c_{0}=0$ and $c_{0}=-\mathscr{L}(x)$, and even

$$
\frac{x(T)}{f(T, x(T))}+c_{0}+c_{1} T=\int_{0}^{T}(T-s)^{\alpha-1} h(s) d s .
$$

Thus,

$$
c_{1}=\frac{1}{T}\left(\mathscr{L}(x)-x_{T}+\frac{1}{\Gamma(\alpha)} \int_{0}^{T}(T-s)^{\alpha-1} h(s) d s\right)
$$

implies that

$$
\begin{aligned}
\frac{x(t)}{f(t, x(t))}= & \frac{1}{\Gamma(\alpha)} \int_{0}^{t}(t-s)^{\alpha-1} h(s) d s \\
& -\frac{t}{T \Gamma(\alpha)} \int_{0}^{T}(T-s)^{\alpha-1} h(s) d s \\
& -\left(\frac{t}{T}-1\right) \mathscr{L}(x)+\frac{t}{T} x_{T} .
\end{aligned}
$$


Theorem 8. Assume hypotheses $\left(H_{0}\right)-\left(H_{3}\right)$. Further, if

$$
L\left(\frac{2 T^{\alpha}}{\Gamma(\alpha+1)}\|h\|_{L^{1}}+M+\left|x_{T}\right|\right)<1,
$$

then the hybrid fractional order differential equation (1) has a solution defined on $J$.

Proof. We defined a subset $S$ of $X$ by

$$
S=\left\{x \in \frac{X}{\|x\|} \leq N\right\},
$$

where $N=F_{0}\left(\left(2 T^{\alpha} / \Gamma(\alpha+1)\right)\|h\|_{L^{1}}+M+\left|x_{T}\right|\right) /(1-$ $\left.L\left(\left(2 T^{\alpha} / \Gamma(\alpha+1)\right)\|h\|_{L^{1}}+M+\left|x_{T}\right|\right)\right)$ and $F_{0}=\sup _{t \in J}|f(t, 0)|$.

It is clear that $S$ satisfies hypothesis of Lemma 6 . By an application of Lemma 7, (1) is equivalent to the nonlinear hybrid integral equation

$$
\begin{aligned}
x(t) & =f(t, x(t))\left[\frac{1}{\Gamma(\alpha)} \int_{0}^{t}(t-s)^{\alpha-1} g(s, x(s)) d s\right. \\
& -\frac{t}{T \Gamma(\alpha)} \int_{0}^{T}(T-s)^{\alpha-1} g(s, x(s)) d s \\
& \left.-\left(\frac{t}{T}-1\right) \mathscr{L}(x)+\frac{t}{T} x_{T}\right] .
\end{aligned}
$$

$$
\begin{aligned}
& -\frac{t}{T \Gamma(\alpha)} \int_{0}^{T}(T-s)^{\alpha-1} g(s, x(s)) d s \\
& -\left(\frac{t}{T}-1\right) \mathscr{L}(x)+\frac{t}{T} x_{T} .
\end{aligned}
$$

Then the hybrid integral equation (25) is transformed into the operator equation as

$$
x(t)=A x(t) B x(t), \quad t \in J
$$

We will show that the operators $A$ and $B$ satisfy all the conditions of Lemma 6.

Claim 1. Let $x, y \in X$. Then by hypothesis $\left(\mathrm{H}_{1}\right)$,

$$
\begin{aligned}
|A x(t)-A y(t)| & =|f(t, x(t))-f(t, y(t))| \\
& \leq L|x(t)-y(t)| \leq L\|x-y\|,
\end{aligned}
$$

for all $t \in J$. Taking supremum over $t$, we obtain

$$
\|A x-A y\| \leq L\|x-y\|,
$$

for all $x, y \in X$.
Claim 2 (we show that $B$ is continuous in $S$ ). Let $\left(x_{n}\right)$ be a sequence in $S$ converging to a point $x \in S$. Then by Lebesgue dominated convergence theorem,

$$
\begin{aligned}
& \lim _{n \rightarrow \infty} \frac{1}{\Gamma(\alpha)} \int_{0}^{t}(t-s)^{\alpha-1} g\left(s, x_{n}(s)\right) d s \\
&= \frac{1}{\Gamma(\alpha)} \int_{0}^{t}(t-s)^{\alpha-1} \lim _{n \rightarrow \infty} g\left(s, x_{n}(s)\right) d s, \\
& \lim _{n \rightarrow \infty} \frac{b}{\Gamma(\alpha)} \int_{0}^{T}(T-s)^{\alpha-1} g\left(s, x_{n}(s)\right) d s \\
&=\frac{b}{\Gamma(\alpha)} \int_{0}^{T}(T-s)^{\alpha-1} \lim _{n \rightarrow \infty} g\left(s, x_{n}(s)\right) d s .
\end{aligned}
$$

And since $\mathscr{L}$ is a continuous function

$$
\lim _{n \rightarrow \infty} \mathscr{L}\left(x_{n}\right)=\mathscr{L}(x)
$$

then

$$
\begin{aligned}
& \lim _{n \rightarrow \infty} B x_{n}(t)=\lim _{n \rightarrow \infty}\left[\frac{1}{\Gamma(\alpha)} \int_{0}^{t}(t-s)^{\alpha-1} g\left(s, x_{n}(s)\right) d s\right. \\
& -\frac{t}{T \Gamma(\alpha)} \int_{0}^{T}(T-s)^{\alpha-1} g\left(s, x_{n}(s)\right) d s \\
& \left.-\left(\frac{t}{T}-1\right) \mathscr{L}\left(x_{n}\right)+\frac{t}{T} x_{T}\right]=\lim _{n \rightarrow \infty} \frac{1}{\Gamma(\alpha)} \\
& \cdot \int_{0}^{t}(t-s)^{\alpha-1} g\left(s, x_{n}(s)\right) d s-\lim _{n \rightarrow \infty} \frac{t}{T \Gamma(\alpha)} \\
& \cdot \int_{0}^{T}(T-s)^{\alpha-1} g\left(s, x_{n}(s)\right) d s-\lim _{n \rightarrow \infty}\left(\frac{t}{T}-1\right) \\
& \cdot \mathscr{L}\left(x_{n}\right)+\frac{t}{T} x_{T}=\frac{1}{\Gamma(\alpha)} \int_{0}^{t}(t-s)^{\alpha-1} g(s, x(s)) d s \\
& -\frac{t}{T \Gamma(\alpha)} \int_{0}^{T}(T-s)^{\alpha-1} g(s, x(s)) d s-\left(\frac{t}{T}-1\right) \\
& \cdot \mathscr{L}(x)+\frac{t}{T} x_{T}=B x(t),
\end{aligned}
$$

for all $t \in J$. This shows that $B$ is a continuous operator on $S$.

Claim 3 ( $B$ is compact operator on $S$ ). First, we show that $B(S)$ is a uniformly bounded set in $X$.

Let $x \in S$. Then by hypothesis $\left(\mathrm{H}_{2}\right)$, for all $t \in J$,

$$
\begin{aligned}
|B x(t)| \leq & \frac{1}{\Gamma(\alpha)} \int_{0}^{t}\left|(t-s)^{\alpha-1} g(s, x(s))\right| d s \\
& +\frac{1}{\Gamma(\alpha)} \int_{0}^{T}\left|(T-s)^{\alpha-1} g(s, x(s))\right| d s \\
& +|\mathscr{L}(x)|+\left|x_{T}\right| \\
\leq & \frac{T^{\alpha}}{\alpha \Gamma(\alpha)}\|h\|_{L^{1}}+\frac{T^{\alpha}}{\alpha \Gamma(\alpha)}\|h\|_{L^{1}}+|\mathscr{L}(x)| \\
& +\left|x_{T}\right| \leq \frac{2 T^{\alpha}}{\Gamma(\alpha+1)}\|h\|_{L^{1}}+M+\left|x_{T}\right| .
\end{aligned}
$$

Thus, $\|B x\| \leq\left(2 T^{\alpha} / \Gamma(\alpha+1)\right)\|h\|_{L^{1}}+M+\left|x_{T}\right|$, for all $x \in S$. 
This shows that $B$ is uniformly bounded on $S$.

Next, we show that $B(S)$ is an equicontinuous set on $X$.

We set $p(t)=\int_{0}^{t} h(s) d s$.

Let $t_{1}, t_{2} \in J$. Then for any $x \in S$,

$$
\begin{aligned}
& \left|B x\left(t_{1}\right)-B x\left(t_{2}\right)\right| \\
& =\mid \frac{1}{\Gamma(\alpha)} \int_{0}^{t_{1}}\left(t_{1}-s\right)^{\alpha-1} g(s, x(s)) d s \\
& -\frac{1}{\Gamma(\alpha)} \int_{0}^{t_{2}}\left(t_{2}-s\right)^{\alpha-1} g(s, x(s)) d s \\
& -\left(t_{1}-t_{2}\right) \frac{1}{T \Gamma(\alpha)} \int_{0}^{T}\left|(T-s)^{\alpha-1} g(s, x(s))\right| d s \\
& -\frac{1}{T}\left(t_{1}-t_{2}\right) \mathscr{L}(x)+\frac{1}{T}\left(t_{1}-t_{2}\right) x_{T} \mid \\
& \leq \frac{T^{\alpha-1}}{\Gamma(\alpha)}\left|\int_{t_{1}}^{t_{2}}\right| g(s, x(s))|d s|+\frac{T^{\alpha-1}}{\Gamma(\alpha)}\left|t_{1}-t_{2}\right| \\
& . \int_{0}^{T}|g(s, x(s))| d s+\frac{M+\left|x_{T}\right|}{T}\left|t_{1}-t_{2}\right| \\
& \quad \leq \frac{T^{\alpha-1}}{\Gamma(\alpha)}\left|p\left(t_{1}\right)-p\left(t_{2}\right)\right|+\left(\frac{T^{\alpha-1}}{\Gamma(\alpha)}+\frac{M+\left|x_{T}\right|}{T}\right) \\
& \cdot\left|t_{1}-t_{2}\right| .
\end{aligned}
$$

Since $p$ is continuous on compact $J$, it is uniformly continuous. Hence,

$$
\begin{aligned}
\left|t_{1}-t_{2}\right|<\eta \Longrightarrow\left|B x\left(t_{1}\right)-B x\left(t_{2}\right)\right|<\varepsilon, & \\
& \forall \varepsilon>0, \quad \exists \eta>0
\end{aligned}
$$

for all $t_{1}, t_{2} \in J$ and for all $x \in X$.

This shows that $B(S)$ is an equicontinuous set in $X$.

Then by Arzelá-Ascoli theorem, $B$ is a continuous and compact operator on $S$.

Claim 4 (hypothesis (c) of Lemma 6 is satisfied). Let $x \in X$ and $y \in S$ be arbitrary such that $x=A x B y$. Then,

$$
\begin{gathered}
|x(t)|=|A x(t)||B y(t)| \leq|f(t, x(t))| \\
\cdot \mid \frac{1}{\Gamma(\alpha)} \int_{0}^{t}(t-s)^{\alpha-1} g(s, x(s)) d s \\
-\frac{t}{T \Gamma(\alpha)} \int_{0}^{T}(T-s)^{\alpha-1} g(s, x(s)) d s \\
-\left(\frac{t}{T}-1\right) \mathscr{L}(x)+\frac{t}{T} x_{T} \mid
\end{gathered}
$$

$$
\begin{aligned}
& \leq[|f(t, x(t))-f(t, 0)|+|f(t, 0)|] \\
& \cdot\left(\frac{2 T^{\alpha}}{\Gamma(\alpha+1)}\|h\|_{L^{1}}+M+\left|x_{T}\right|\right) \leq\left(L|x(t)|+F_{0}\right) \\
& \cdot\left(\frac{2 T^{\alpha}}{\Gamma(\alpha+1)}\|h\|_{L^{1}}+M+\left|x_{T}\right|\right)
\end{aligned}
$$

and so,

$$
\begin{aligned}
& |x(t)|-L\left(\frac{2 T^{\alpha}}{\Gamma(\alpha+1)}\|h\|_{L^{1}}+M+\left|x_{T}\right|\right)|x(t)| \\
& \leq F_{0}\left(\frac{2 T^{\alpha}}{(\alpha+1)}\|h\|_{L^{1}}+M+\left|x_{T}\right|\right),
\end{aligned}
$$

which implies

$$
|x(t)| \leq \frac{F_{0}\left(\left(2 T^{\alpha} / \Gamma(\alpha+1)\right)\|h\|_{L^{1}}+M+\left|x_{T}\right|\right)}{1-L\left(\left(2 T^{\alpha} / \Gamma(\alpha+1)\right)\|h\|_{L^{1}}+M+\left|x_{T}\right|\right)} .
$$

Taking supremum over $t$,

$$
\begin{aligned}
\|x\| & \leq \frac{F_{0}\left(\left(2 T^{\alpha} / \Gamma(\alpha+1)\right)\|h\|_{L^{1}}+M+\left|x_{T}\right|\right)}{1-L\left(\left(2 T^{\alpha} / \Gamma(\alpha+1)\right)\|h\|_{L^{1}}+M+\left|x_{T}\right|\right)} \\
& =N .
\end{aligned}
$$

Then $x \in S$, and hypothesis (c) of Lemma 6 is satisfied.

Finally, we have

$$
\begin{aligned}
M & =\|B(S)\|=\sup \{\|B x\|: x \in S\} \\
& \leq \frac{2 T^{\alpha}}{\Gamma(\alpha+1)}\|h\|_{L^{1}}+M+\left|x_{T}\right| .
\end{aligned}
$$

So,

$$
\alpha M \leq L\left(\frac{2 T^{\alpha}}{\Gamma(\alpha+1)}\|h\|_{L^{1}}+M+\left|x_{T}\right|\right)<1 .
$$

Thus, all the conditions of Lemma 6 are satisfied and hence the operator equation $A x B x=x$ has a solution in $S$. As a result, BVPHDEFNL (1) has a solution defined on $J$. This completes the proof.

\section{An Example}

In this section we give an example to illustrate the usefulness of our main results. Let us consider the following fractional boundary value problem:

$$
\begin{aligned}
& { }^{c} D^{\alpha}\left(\frac{(2+\ln (t+1))\left(x(t)+x^{2}(t)\right)}{e^{1-t}}\right) \\
& =\frac{e^{-x^{2}(t)}}{x^{2}(t)+t^{2}+1} \quad \text { a.e. } t \in J=[0,1], 1<\alpha \leq 2, \\
& \frac{x(0)}{f(0, x(0))}=\sum_{i=1}^{n} c_{i} x\left(t_{i}\right) \\
& \frac{x(1)}{f(1, x(1))}=1
\end{aligned}
$$


where $0<t_{1}<t_{2}<\cdots<t_{n}<1, c_{i}, i=1,2, \ldots, n$, are given positive constants.

$$
\begin{aligned}
& \text { And } \sum_{i=1}^{n} c_{i}<(1-\pi) / 2 \bar{M} \text {, where } \bar{M}=\max _{1 \leq i \leq n} \mathscr{L}\left(t_{i}\right) . \\
& \text { We set } \\
& f(t, x)=\frac{e^{t-1}}{(2+\ln (1+t))(1+x)}, \\
& \qquad(t, x) \in[0,1] \times[0,+\infty), \\
& \mathscr{L}(t, x)=\frac{e^{-x^{2}}}{x^{2}+t^{2}+1}, \\
& \mathscr{L}(t))=\sum_{i=1}^{n} c_{i} x\left(t_{i}\right) .
\end{aligned}
$$

Let $x, y \in[0,+\infty)$ and $t \in J$. We have

$$
\begin{aligned}
|f(t, x)-f(t, y)| & =\frac{e^{t-1}}{2+\ln (1+t)}\left|\frac{1}{1+x}-\frac{1}{1+y}\right| \\
& \leq \frac{1}{2}\left|\frac{x-y}{(1+x)(1+y)}\right| \\
& \leq \frac{1}{2}|x-y| .
\end{aligned}
$$

Hence, condition $\left(\mathrm{H}_{1}\right)$ holds with $L=1 / 2$. Also we have

$$
\begin{aligned}
|g(t, x)| & =\frac{e^{-x^{2}}}{x^{2}+t^{2}+1} \\
& \leq h(t),
\end{aligned}
$$

where $h(t)=1 /\left(1+t^{2}\right)$. We have

$$
\int_{0}^{1} h(t) d t=\int_{0}^{1} \frac{1}{1+t^{2}}=\frac{\pi}{4} .
$$

Then condition $\left(\mathrm{H}_{2}\right)$ holds.

Furthermore, since $\mathscr{L} \in C(J, \mathbb{R})$, then we set $\bar{M}=$ $\max _{1 \leq i \leq n} \mathscr{L}\left(t_{i}\right)$ and we have

$$
\left|\mathscr{L}\left(t_{i}\right)\right|=\left|\sum_{i=1}^{n} c_{i} \mathscr{L}\left(t_{i}\right)\right| \leq \bar{M} \sum_{i=1}^{n} c_{i} .
$$

We will check that condition (23) is satisfied with $T=1$.

Since $\sum_{i=1}^{n} c_{i}<(1-\pi) / 2 \bar{M}$, then $\pi+2 \bar{M} \sum_{i=1}^{n} c_{i}<1$.

Thus,

$$
\frac{\pi}{\Gamma(\alpha+1)}+2 \bar{M} \sum_{i=1}^{n} c_{i}<1,
$$

which is satisfied for each $\alpha \in(1,2]$. Then by Theorem 8 problem (42) has a solution on $[0,1]$.

\section{System of Coupled Hybrid Fractional Differential Equations}

The aim of this section is to obtain the existence results, by means of Banach's fixed point theorem, for the problem of coupled hybrid fractional differential equations for (1). Consider

$$
\begin{aligned}
{ }^{c} D^{\alpha}\left(\frac{x(t)}{f_{1}(t, x(t), y(t))}\right) & =g_{1}(t, x(t), y(t)) \\
& \text { a.e. } t \in[0,1], 1<\alpha \leq 2, \\
{ }^{c} D^{\beta}\left(\frac{y(t)}{f_{2}(t, x(t), y(t))}\right) & =g_{2}(t, x(t), y(t)) \\
& \text { a.e. } t \in[0,1], 1<\beta \leq 2, \\
\frac{x(0)}{f_{1}(0, x(0), y(0))} & =\mathscr{L}_{1}(x, y), \quad x(1)=0, \\
\frac{y(0)}{f_{2}(0, x(0), y(0))} & =\mathscr{L}_{2}(x, y), \quad y(1)=0,
\end{aligned}
$$

where ${ }^{c} D^{\alpha}$ is the Caputo fractional derivative.

$f_{i} \in C(J \times \mathbb{R} \times \mathbb{R}, \mathbb{R} \backslash\{0\}), g_{i} \in C([0,1] \times \mathbb{R} \times \mathbb{R}, \mathbb{R})$, and $\mathscr{L}_{i}: C([0,1], \mathbb{R}) \times C([0,1], \mathbb{R}) \rightarrow \mathbb{R}$ are continuous functions $(i=1,2)$.

Main Results. Let $\Omega=\left\{\omega(t) \backslash \omega(t) \in C^{1}([0,1])\right\}$ denote a Banach space equipped with the norm $\|\omega\|=\sup \{|\omega(t)|, t \in$ $[0,1]\}$, where $\Omega=\mathscr{K} \times \mathscr{R}$. Notice that the product space $(\mathscr{K} \times \mathscr{R},\|(x, y)\|)$ with the norm $\|(x, y)\|=\|x\|+\|y\|,(x, y) \in$ $\mathscr{K} \times \mathscr{R}$ is also a Banach space.

In view of Lemma 7, we define an operator $\Phi: \mathscr{K} \times \mathscr{R} \rightarrow$ $\mathscr{K} \times \mathscr{R}$ by

$$
\Phi(x, y)(t)=\left(\Phi_{1}(x, y)(t), \Phi_{2}(x, y)(t)\right),
$$

where

$$
\begin{aligned}
& \Phi_{1}(x, y)(t)=f_{1}(t, x(t), y(t)) \\
& \cdot\left[\frac{1}{\Gamma(\alpha)} \int_{0}^{t}(t-s)^{\alpha-1} g_{1}(s, x(s), y(s)) d s\right. \\
& -\frac{t}{\Gamma(\alpha)} \int_{0}^{1}(1-s)^{\alpha-1} g_{1}(s, x(s), y(s)) d s \\
& \left.-(t-1) \mathscr{L}_{1}(x, y)\right], \\
& \Phi_{2}(x, y)(t)=f_{2}(t, x(t), y(t)) \\
& \cdot\left[\frac{1}{\Gamma(\beta)} \int_{0}^{t}(t-s)^{\beta-1} g_{2}(s, x(s), y(s)) d s\right. \\
& -\frac{t}{\Gamma(\beta)} \int_{0}^{1}(1-s)^{\beta-1} g_{2}(s, x(s), y(s)) d s \\
& \left.-(t-1) \mathscr{L}_{2}(x, y)\right] .
\end{aligned}
$$


In the sequel, we need the following assumptions:

$\left(\mathrm{H}_{1}^{\prime}\right)$ : the functions $f_{i}$ are continuous and bounded; that is, there exist positive numbers $L_{i}$ such that $\left|f_{i}(t, u, v)\right| \leq$ $L_{i}$ for all $(t, u, v) \in[0,1] \times \mathbb{R} \times \mathbb{R}(i=1,2)$.

$\left(\mathrm{H}_{2}^{\prime}\right)$ : there exist real constants $\rho_{0}, \delta_{0}>0$ and $\rho_{i}, \delta_{i} \geq 0(i=$ $1,2)$ such that $\left|g_{1}(t, x, y)\right| \leq \rho_{0}+\rho_{1}|x|+\rho_{2}|y|$ and $\left|g_{2}(t, x, y)\right| \leq \delta_{0}+\delta_{1}|x|+\delta_{2}|y|$ for all $x, y \in \mathbb{R}(i=$ $1,2)$.

$\left(\mathrm{H}_{3}^{\prime}\right)$ : there exist real constants $M_{1}, M_{2}>0\left|\mathscr{L}_{1}(x, y)\right| \leq$ $M_{1}$ and $\left|\mathscr{L}_{2}(x, y)\right| \leq M_{2}$ for each $x, y \in C([0,1])$.

$\left(\mathrm{H}_{4}^{\prime}\right)$ : there exist real constants $\gamma_{1}, \gamma, \gamma_{1}^{\prime}, \gamma_{2}^{\prime}$ such that $\left|\mathscr{L}_{1}\left(x_{1}, y_{1}\right)-\mathscr{L}_{1}\left(x_{2}, y_{2}\right)\right| \leq \gamma_{1}\left|x_{1}-x_{2}\right|+\gamma_{2}\left|y_{1}-y_{2}\right|$ and $\left|\mathscr{L}_{2}\left(x_{1}, y_{1}\right)-\mathscr{L}_{2}\left(x_{2}, y_{2}\right)\right| \leq \gamma_{1}^{\prime}\left|x_{1}-x_{2}\right|+\gamma_{2}^{\prime}\left|y_{1}-y_{2}\right|$.

For brevity, let us set

$$
\begin{aligned}
& \mu_{1}=\frac{2 L_{1}}{\Gamma(\alpha+1)}, \\
& \mu_{2}=\frac{2 L_{2}}{\Gamma(\beta+1)}, \\
& \mu_{0}=\min \left\{1-\left(\mu_{1} \rho_{1}+\mu_{2} \delta_{1}\right), 1-\left(\mu_{1} \rho_{2}+\mu_{2} \delta_{2}\right)\right\} .
\end{aligned}
$$

Now we present our result for the existence and uniqueness of solutions for problem (49). This result is based on Banach's contraction mapping principle.

Theorem 9. Suppose that conditions $\left(H_{1}^{\prime}\right),\left(H_{3}^{\prime}\right)$, and $\left(H_{4}^{\prime}\right)$ hold and that $g_{1}, g_{2}:[0,1] \times \mathbb{R}^{2} \rightarrow \mathbb{R}$ are continuous functions. In addition, there exist positive constants $\eta_{i}, \xi_{i}, i=1,2$, such that

$$
\begin{aligned}
& \left|g_{1}\left(t, x_{1}, y_{1}\right)-g_{1}\left(t, x_{2}, y_{2}\right)\right| \\
& =\eta_{1}\left|x_{1}-x_{2}\right|+\eta_{2}\left|y_{1}-y_{2}\right| \text {, } \\
& \left|g_{2}\left(t, x_{1}, y_{1}\right)-g_{2}\left(t, x_{2}, y_{2}\right)\right| \\
& =\xi_{1}\left|x_{1}-x_{2}\right|+\xi_{2}\left|y_{1}-y_{2}\right|, \\
& \forall t \in[0,1], x_{1}, x_{2}, y_{1}, y_{2} \in \mathbb{R} .
\end{aligned}
$$

If $\mu_{1}\left(\eta_{1}+\eta_{2}\right)+\mu_{2}\left(\xi_{1}+\xi_{2}\right)+L_{1}\left(\gamma_{1}+\gamma_{2}\right)+L_{2}\left(\gamma_{1}^{\prime}+\gamma_{2}^{\prime}\right)<1$, then problem (49) has a unique solution.

Proof. Let us set $\sup _{t \in[0,1]} g_{1}(t, 0,0)=\kappa_{1}<\infty$ and $\sup _{t \in[0,1]} g_{2}(t, 0,0)=\kappa_{2}<\infty$ and define a closed ball: $B_{r}=$ $\{(x, y) \in \mathscr{K} \times \mathscr{R}:\|(x, y)\| \leq r\}$, where

$$
r \geq \frac{\mu_{1} \kappa_{1}+\mu_{2} \kappa_{2}+M_{1} L_{1}+M_{2} L_{2}}{1-\mu_{1}\left(\eta_{1}+\eta_{2}\right)-\mu_{2}\left(\xi_{1}+\xi_{2}\right)} .
$$

Claim 5 (we show that $\Phi B_{r} \subset B_{r}$ ). Let $(x, y) \in B_{r}$. We have

$$
\begin{aligned}
& \left|\Phi_{1}(x, y)(t)\right| \leq M_{1}\left[\operatorname { s u p } _ { t \in [ 0 , 1 ] } \left\{\frac{1}{\Gamma(\alpha)} \int_{0}^{t}(t-s)^{\alpha-1}\right.\right. \\
& \cdot\left|g_{1}(s, x(s), y(s))\right| d s+\frac{1}{\Gamma(\alpha)} \int_{0}^{1}(1-s)^{\alpha-1} \\
& \left.\left.\cdot\left|g_{1}(s, x(s), y(s))\right| d s\right\}+\left|\mathscr{L}_{1}(x, y)\right|\right] \\
& =M_{1}\left[\operatorname { s u p } _ { t \in [ 0 , 1 ] } \left\{\frac{1}{\Gamma(\alpha)} \int_{0}^{t}(t-s)^{\alpha-1}\right.\right. \\
& \cdot\left(\left|g_{1}(s, x(s), y(s))-g_{1}(s, 0,0)\right|+\left|g_{1}(s, 0,0)\right|\right) d s \\
& +\frac{1}{\Gamma(\alpha)} \int_{0}^{1}(t-s)^{\alpha-1} \\
& \left.\cdot\left(\left|g_{1}(s, x(s), y(s))-g_{1}(s, 0,0)\right|+\left|g_{1}(s, 0,0)\right|\right) d s\right\} \\
& \left.+\left|\mathscr{L}_{1}(x, y)\right|\right] \leq M_{1}\left[\frac{2}{\Gamma(\alpha+1)}\left(\eta_{1}\|x\|+\eta_{2}\|y\|+\kappa_{1}\right)\right. \\
& \left.+L_{1}\right] \leq M_{1}\left[\frac{2}{\Gamma(\alpha+1)}\left(\left(\eta_{1}+\eta_{2}\right) r+\kappa_{1}\right)+L_{1}\right] \\
& \leq \mu_{1}\left[\left(\eta_{1}+\eta_{2}\right) r+\kappa_{1}\right]+M_{1} L_{1} \cdot
\end{aligned}
$$

Hence,

$$
\begin{gathered}
\left\|\Phi_{1}(x, y)\right\| \leq \mu_{1}\left[\left(\eta_{1}+\eta_{2}\right) r+\kappa_{1}\right]+M_{1} L_{1}, \\
\left\|\Phi_{2}(x, y)\right\| \leq \mu_{2}\left[\left(\xi_{1}+\xi_{2}\right) r+\kappa_{2}\right]+M_{2} L_{2} .
\end{gathered}
$$

From (57), it follows that $\|\Phi(x, y)\| \leq r$.

Next, for $\left(x_{1}, y_{1}\right),\left(x_{2}, y_{2}\right) \in \mathscr{K} \times \mathscr{R}$ and for any $t \in[0,1]$, we have

$$
\begin{aligned}
& \left|\Phi_{1}\left(x_{2}, y_{2}\right)(t)-\Phi_{1}\left(x_{1}, y_{1}\right)(t)\right| \\
& \quad \leq L_{1}\left[\int_{0}^{t} \frac{(t-s)^{\alpha-1}}{\Gamma(\alpha)} \mid g_{1}\left(s, x_{2}(s), y_{2}(s)\right)\right. \\
& \quad-g_{1}\left(s, x_{1}(s), y_{1}(s)\right) \mid d s \\
& \quad+\int_{0}^{1} \frac{(1-s)^{\alpha-1}}{\Gamma(\alpha)} \mid g_{1}\left(s, x_{2}(s), y_{2}(s)\right) \\
& -g_{1}\left(s, x_{1}(s), y_{1}(s)\right)|d s+| \mathscr{L}_{1}\left(x_{2}, y_{2}\right) \\
& \left.-\mathscr{L}_{1}\left(x_{1}, y_{1}\right) \mid\right] \leq L_{1}\left[\frac { 2 } { \Gamma ( \alpha + 1 ) } \left(\eta_{1} \mid x_{2}(t)\right.\right. \\
& \left.-x_{1}(t)\left|+\eta_{2}\right| y_{2}(t)-y_{1}(t) \mid\right)+\gamma_{1}\left|x_{2}(t)-x_{1}(t)\right| \\
& \left.+\gamma_{2}\left|y_{2}(t)-y_{1}(t)\right|\right] \leq \mu_{1}\left(\eta_{1}\left\|x_{2}-x_{1}\right\|+\eta_{2} \| y_{2}\right. \\
& \left.-y_{1} \|\right)+L_{1}\left(\gamma_{1}\left\|x_{2}-x_{1}\right\|+\gamma_{2}\left\|y_{2}-y_{1}\right\|\right) \\
& \quad \leq\left[\mu_{1}\left(\eta_{1}+\eta_{2}\right)+L_{1}\left(\gamma_{1}+\gamma_{2}\right)\right]\left(\left\|x_{2}-x_{1}\right\|+\| y_{2}\right. \\
& \left.-y_{1} \|\right)
\end{aligned}
$$


which yields

$$
\begin{aligned}
& \left\|\Phi_{1}\left(x_{2}, y_{2}\right)-\Phi_{1}\left(x_{1}, y_{1}\right)\right\| \\
& \quad \leq\left[\mu_{1}\left(\eta_{1}+\eta_{2}\right)+L_{1}\left(\gamma_{1}+\gamma_{2}\right)\right] \\
& \cdot\left(\left\|x_{2}-x_{1}\right\|+\left\|y_{2}-y_{1}\right\|\right) .
\end{aligned}
$$

Working in a similar manner, one can find that

$$
\begin{aligned}
& \left\|\Phi_{2}\left(x_{2}, y_{2}\right)-\Phi_{2}\left(x_{1}, y_{1}\right)\right\| \\
& \quad \leq\left[\mu_{2}\left(\xi_{1}+\xi_{2}\right)+L_{2}\left(\gamma_{1}^{\prime}+\gamma_{2}^{\prime}\right)\right] \\
& \cdot\left(\left\|x_{2}-x_{1}\right\|+\left\|y_{2}-y_{1}\right\|\right) .
\end{aligned}
$$

We deduce that

$$
\begin{aligned}
& \left\|\Phi\left(x_{2}, y_{2}\right)-\Phi\left(x_{1}, y_{1}\right)\right\| \leq\left[\mu_{1}\left(\eta_{1}+\eta_{2}\right)\right. \\
& \left.+\mu_{2}\left(\xi_{1}+\xi_{2}\right)+L_{1}\left(\gamma_{1}+\gamma_{2}\right)+L_{2}\left(\gamma_{1}^{\prime}+\gamma_{2}^{\prime}\right)\right] \\
& \quad \cdot\left(\left\|x_{2}-x_{1}\right\|+\left\|y_{2}-y_{1}\right\|\right) .
\end{aligned}
$$

In view of condition $\mu_{1}\left(\eta_{1}+\eta_{2}\right)+\mu_{2}\left(\xi_{1}+\xi_{2}\right)+L_{1}\left(\gamma_{1}+\gamma_{2}\right)+$ $L_{2}\left(\gamma_{1}^{\prime}+\gamma_{2}^{\prime}\right)<1$, it follows that $\Phi$ is a contraction. So $\Phi$ has a unique fixed point. This implies that problem (49) has a unique solution on $[0,1]$. This completes the proof.

In our second result, we discuss the existence of solutions for problem (49) by means of Leray-Schauder alternative.

Lemma 10 (see [17]). Let $\mathfrak{F}: \mathfrak{I} \rightarrow \mathfrak{I}$ be a completely continuous operator (i.e., a map that is restricted to any bounded set in $G$ is compact). Let $\mathscr{P}(\mathfrak{F})=\{x \in \mathfrak{J}: x=$ $\lambda \mathfrak{F} x$ for some $0<\lambda<1\}$. Then either the set $\mathscr{P}(\mathfrak{F})$ is unbounded or $\mathfrak{F}$ has at least one fixed point.

Theorem 11. Assume that conditions $\left(H_{1}^{\prime}\right)-\left(H_{3}^{\prime}\right)$ hold. Furthermore, it is assumed that $\mu_{1} \rho_{1}+\mu_{2} \delta_{1}<1$ and $\mu_{1} \rho_{2}+\mu_{2} \delta_{2}<$ 1 , where $\mu_{1}$ and $\mu_{2}$ are given by (52). Then the boundary value problem (49) has at least one solution.

Proof. We will show that the operator $\Phi: \mathscr{K} \times \mathscr{R} \rightarrow \mathscr{K} \times \mathscr{R}$ satisfies all the assumptions of Lemma 10. In the first step, we prove that the operator $\Phi$ is completely continuous. Clearly, it follows by the continuity of functions $f_{1}, f_{2}, g_{1}$, and $g_{2}$ that the operator $\Phi$ is continuous.

Let $\mathcal{S} \subset \mathscr{K} \times \mathscr{R}$ be bounded. Then we can find positive constants $N_{1}$ and $N_{2}$ such that

$$
\begin{aligned}
\left|g_{1}(t, x(t), y(t))\right| & \leq N_{1} \\
\left|g_{2}(t, x(t), y(t))\right| & \leq N_{2}, \\
\forall(x, y) & \in \mathcal{S} .
\end{aligned}
$$

Thus, for any $x, y \in \mathcal{S}$, we can get

$$
\begin{aligned}
& \left|\Phi_{1}(x, y)(t)\right| \\
& \quad \leq L_{1}\left[\int_{0}^{t} \frac{(t-s)^{\alpha-1}}{\Gamma(\alpha)}\left|g_{1}(s, x(s), y(s))\right| d s\right. \\
& \quad+t \int_{0}^{1} \frac{(1-s)^{\beta-1}}{\Gamma(\beta)}\left|g_{1}(s, x(s), y(s))\right| d s \\
& \left.\quad+(1-t) \mathscr{L}_{1}(x, y)\right] \leq L_{1} N_{1} \frac{2}{\Gamma(\alpha+1)}+M_{1}
\end{aligned}
$$

which yields

$$
\left\|\Phi_{1}(x, y)\right\|=N_{1} \mu_{1}+M_{1} .
$$

In a similar manner,

$$
\left\|\Phi_{2}(x, y)\right\|=N_{2} \mu_{2}+M_{2} .
$$

We deduce that the operator $\Phi$ is uniformly bounded.

Now we show that the operator $\Phi$ is equicontinuous.

We take $\tau_{1}, \tau_{2} \in[0,1]$ with $\tau_{1}<\tau_{2}$ and obtain

$$
\begin{aligned}
& \left|\Phi_{1}\left(x\left(\tau_{2}\right), y\left(\tau_{2}\right)\right)-\Phi_{1}\left(x\left(\tau_{1}\right), y\left(\tau_{1}\right)\right)\right| \\
& \leq L_{1} N_{1}\left|\int_{0}^{\tau_{2}} \frac{\left(\tau_{2}-s\right)^{\alpha-1}}{\Gamma(\alpha)} d s-\int_{0}^{\tau_{1}} \frac{\left(\tau_{1}-s\right)^{\alpha-1}}{\Gamma(\alpha)} d s\right| \\
& +L_{1} N_{1}\left|\tau_{2}-\tau_{1}\right| \int_{0}^{1} \frac{(1-s)^{\alpha-1}}{\Gamma(\alpha)} d s+M_{1}\left|\tau_{2}-\tau_{1}\right| \\
& \leq L_{1} N_{1} \mid \int_{0}^{\tau_{1}} \frac{\left(\tau_{1}-s\right)^{\alpha-1}-\left(\tau_{2}-s\right)^{\alpha-1}}{\Gamma(\alpha)} d s \\
& -\int_{\tau_{1}}^{\tau_{2}} \frac{\left(\tau_{2}-s\right)^{\alpha-1}}{\Gamma(\alpha)} d s\left|+L_{1} N_{1}\right| \tau_{2}-\tau_{1} \mid \\
& \cdot \int_{0}^{1} \frac{(1-s)^{\alpha-1}}{\Gamma(\alpha)} d s+M_{1}\left|\tau_{2}-\tau_{1}\right| \longrightarrow_{\tau_{1} \rightarrow \tau_{2}} 0, \\
& \left|\Phi_{2}\left(x\left(\tau_{2}\right), y\left(\tau_{2}\right)\right)-\Phi_{2}\left(x\left(\tau_{1}\right), y\left(\tau_{1}\right)\right)\right| \\
& \leq L_{2} N_{2}\left|\int_{0}^{\tau_{2}} \frac{\left(\tau_{2}-s\right)^{\beta-1}}{\Gamma(\beta)} d s-\int_{0}^{\tau_{1}} \frac{\left(\tau_{1}-s\right)^{\beta-1}}{\Gamma(\beta)} d s\right| \\
& +L_{2} N_{2}\left|\tau_{2}-\tau_{1}\right| \int_{0}^{1} \frac{(1-s)^{\beta-1}}{\Gamma(\beta)} d s+M_{2}\left|\tau_{2}-\tau_{1}\right| \\
& \leq L_{2} N_{2} \mid \int_{0}^{\tau_{1}} \frac{\left(\tau_{1}-s\right)^{\beta-1}-\left(\tau_{2}-s\right)^{\beta-1}}{\Gamma(\beta)} d s \\
& -\int_{\tau_{1}}^{\tau_{2}} \frac{\left(\tau_{2}-s\right)^{\beta-1}}{\Gamma(\beta)} d s\left|+L_{2} N_{2}\right| \tau_{2}-\tau_{1} \mid \\
& \cdot \int_{0}^{1} \frac{(1-s)^{\beta-1}}{\Gamma(\beta)} d s+M_{2}\left|\tau_{2}-\tau_{1}\right| \longrightarrow_{\tau_{1} \rightarrow \tau_{2}} 0 \text {, }
\end{aligned}
$$


which tend to 0 independently of $(x, y)$. This implies that the operator $\Phi(x, y)$ is equicontinuous. Thus, by the above findings, the operator $\Phi(x, y)$ is completely continuous.

In the next step, it will be established that the set $\mathscr{P}=$ $\{(x, y) \in \mathscr{K} \times \mathscr{R} /(x, y)=\lambda \Phi(x, y), 0 \leq \lambda \leq 1\}$ is bounded.

Let $(x, y) \in \mathscr{P}$. Then we have $(x, y)=\lambda \Phi(x, y)$. Thus, for any $t \in[0,1]$, we can write

$$
\begin{aligned}
& x(t)=\lambda \Phi_{1}(x, y)(t), \\
& y(t)=\lambda \Phi_{2}(x, y)(t) .
\end{aligned}
$$

Then,

$$
\begin{aligned}
& |x(t)| \leq \frac{2 L_{1}}{\Gamma(\alpha+1)}\left(\rho_{0}+\rho_{1}\|x\|+\rho_{2}\|y\|\right)+M_{1}, \\
& |y(t)| \leq \frac{2 L_{2}}{\Gamma(\beta+1)}\left(\delta_{0}+\delta_{1}\|x\|+\delta_{2}\|y\|\right)+M_{2},
\end{aligned}
$$

which imply that

$$
\begin{aligned}
& \|x\| \leq \mu_{1}\left(\rho_{0}+\rho_{1}\|x\|+\rho_{2}\|y\|\right)+M_{1}, \\
& \|y\| \leq \mu_{2}\left(\delta_{0}+\delta_{1}\|x\|+\delta_{2}\|y\|\right)+M_{2} .
\end{aligned}
$$

Thus,

$$
\begin{aligned}
\|x\|+\|y\| \leq & \left(\mu_{1} \rho_{0}+\mu_{2} \delta_{0}+M_{1}+M_{2}\right) \\
& +\left(\mu_{1} \rho_{1}+\mu_{2} \delta_{1}\right)\|x\| \\
& +\left(\mu_{1} \rho_{2}+\mu_{2} \delta_{2}\right)\|y\|,
\end{aligned}
$$

which, in view of (55), gives

$$
\|(x, y)\| \leq \frac{\mu_{1} \rho_{0}+\mu_{2} \delta_{0}+M_{1}+M_{2}}{\mu_{0}} .
$$

This shows that the set is bounded. Hence, all the conditions of Lemma 10 are satisfied and consequently the operator $\Phi$ has at least one fixed point, which corresponds to a solution of problem (49). This completes the proof.

\section{Competing Interests}

The authors declare that there are no competing interests regarding the publication of this paper.

\section{References}

[1] R. P. Agarwal, V. Lakshmikantham, and J. J. Nieto, "On the concept of solution for fractional differential equations with uncertainty," Nonlinear Analysis: Theory, Methods \& Applications, vol. 72, no. 6, pp. 2859-2862, 2010.

[2] L. Byszewski and V. Lakshmikantham, "Theorem about the existence and uniqueness of a solution of a nonlocal abstract Cauchy problem in a Banach space," Applicable Analysis, vol. 40, no. 1, pp. 11-19, 1991.

[3] A. M. El-Sayed, "Fractional order evolution equations," Journal of Fractional Calculus, vol. 7, pp. 89-100, 1995.
[4] A. A. Kilbas, H. M. Srivastava, and J. J. Trujillo, Theory and Applications of Fractional Differential Equations, vol. 204 of North-Holland Mathematics Studies, Elsevier Science B.V., Amsterdam, The Netherlands, 2006.

[5] V. Lakshmikantham and A. S. Vatsala, "Basic theory of fractional differential equations," Nonlinear Analysis: Theory, Methods \& Applications, vol. 69, no. 8, pp. 2677-2682, 2008.

[6] V. Lakshmikantham, "Theory of fractional functional differential equations," Nonlinear Analysis: Theory, Methods \& Applications, vol. 69, no. 10, pp. 3337-3343, 2008.

[7] I. Podlubny, Fractional Differential Equations, vol. 198 of Mathematics in Sciences and Engineering, Academic Press, San Diego, Calif, USA, 1999.

[8] B. C. Dhage, "On a condensing mappings in Banach algebras," The Mathematics Student, vol. 63, no. 1-4, pp. 146-152, 1994.

[9] B. C. Dhage, "A nonlinear alternative in Banach algebras with applications to functional differential equations," Nonlinear Functional Analysis and Applications, vol. 8, pp. 563-575, 2004.

[10] B. C. Dhage, "On a fixed point theorem in Banach algebras with applications," Applied Mathematics Letters, vol. 18, no. 3, pp. 273-280, 2005.

[11] B. C. Dhage and V. Lakshmikantham, "Basic results on hybrid differential equations," Nonlinear Analysis: Hybrid Systems, vol. 4, no. 3, pp. 414-424, 2010.

[12] B. C. Dhage, "Quadratic perturbations of periodic boundary value problems of second order ordinary differential equations," Differential Equations \& Applications, vol. 2, no. 4, pp. 465-486, 2010.

[13] Y. Zhao, S. Sun, Z. Han, and Q. Li, "Theory of fractional hybrid differential equations," Computers and Mathematics with Applications, vol. 62, no. 3, pp. 1312-1324, 2011.

[14] K. Hilal and A. Kajouni, "Boundary value problems for hybrid differential equations with fractional order," Advances in Difference Equations, vol. 2015, article 183, 2015.

[15] K. Ezzinbi, X. Fu, and K. Hilal, "Existence and regularity in the $\alpha$-norm for some neutral partial differential equations with nonlocal conditions," Nonlinear Analysis: Theory, Methods \& Applications, vol. 67, no. 5, pp. 1613-1622, 2007.

[16] S. Zhang, "Positive solutions for boundary value problems of nonlinear fractional differential equations," Electronic Journal of Differential Equations, vol. 36, pp. 1-12, 2006.

[17] A. Granas and J. Dugundji, Fixed Point Theory, Springer, New York, NY, USA, 2003. 


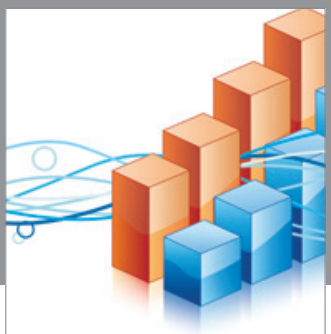

Advances in

Operations Research

vatem alat4

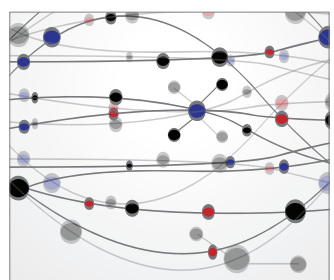

\section{The Scientific} World Journal
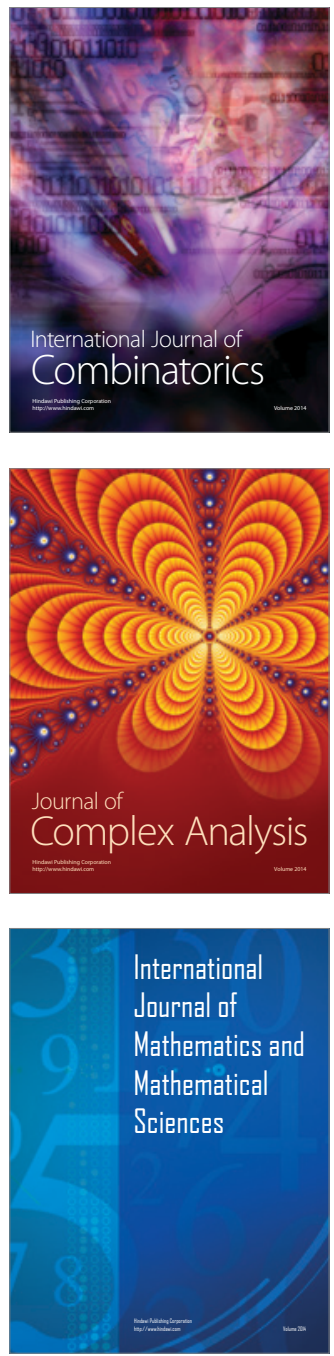
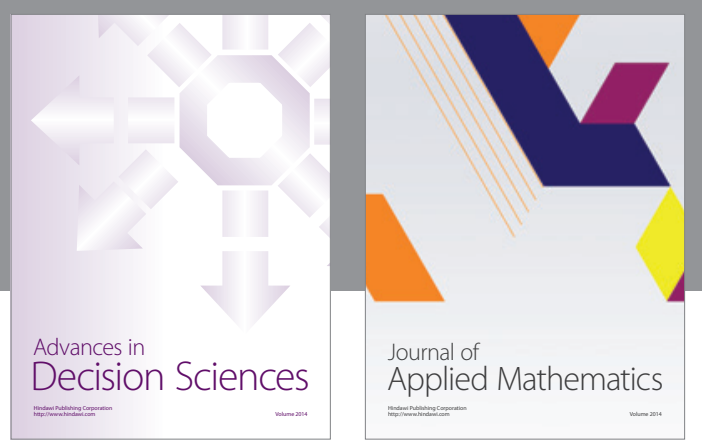

Algebra

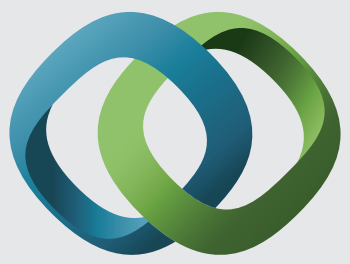

\section{Hindawi}

Submit your manuscripts at

http://www.hindawi.com
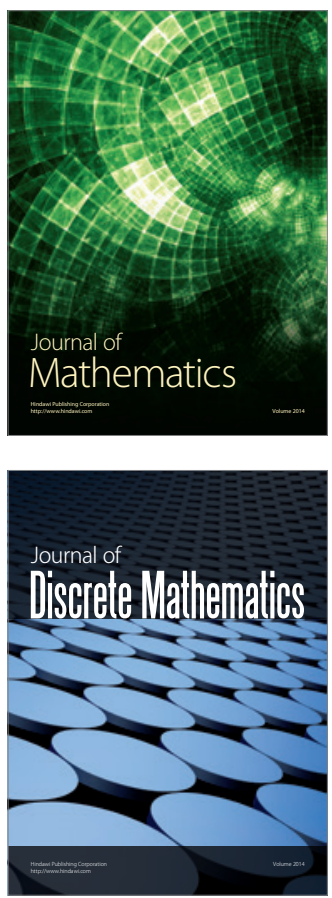

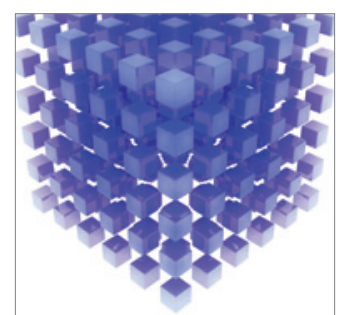

Mathematical Problems in Engineering
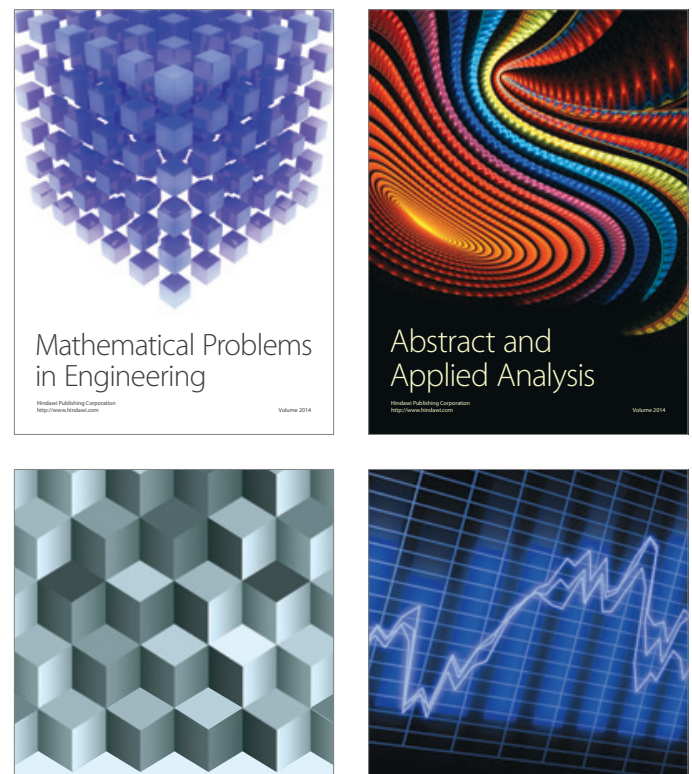

Journal of

Function Spaces

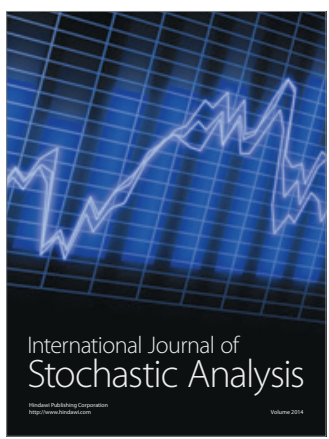

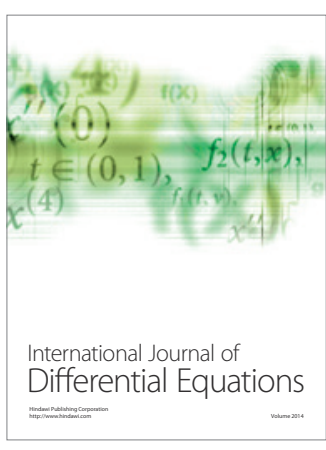
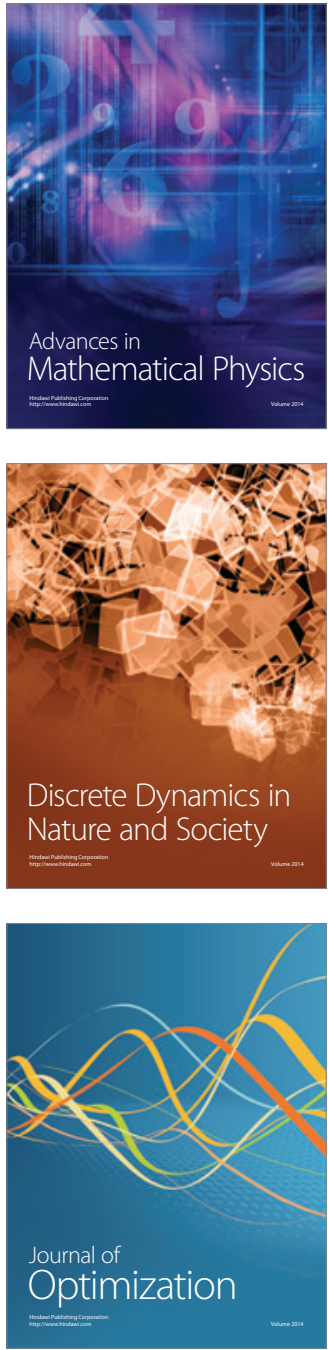